\title{
Airfoil optimization design based on a combined optimization strategy
}

\author{
Pengbao $\mathrm{Ma}^{1, \text { a) }}$, Jianqiao $\mathrm{Yu}^{1)}$, Fangzheng $\mathrm{Chen}^{1)}$ and Zheyu $\mathrm{Xue}^{2)}$ \\ ${ }^{1}$ School of Aerospace Engineering, Beijing Institute of Technology, Beijing 100089, China \\ ${ }^{2}$ School of electrical engineering, Beijing University of Posts and Telecommunications, Beijing \\ 100088, China \\ a)m1346851654@163.com
}

Keywords: Airfoil, Combined optimization

\begin{abstract}
In order to improve the computational efficiency of the airfoil optimization design, a universal algorithm transformation strategy is proposed. The Multi-Island Genetic Algorithm and the Sequential Quadratic Programming are combined to take into account the global search and fast convergence characteristics. The Hicks-Henne airfoil parameterization method is improved to promote the accuracy of the airfoil tail. Based on the Isight optimization design platform, the airfoil optimization system is built up in a modular way. The multi constraint optimization design of RAE2822 airfoil with lift drag ratio as objective function is presented. The experimental results show that the combined optimization algorithm can reduce the computational complexity and improve the efficiency of optimization.
\end{abstract}

\section{INTRODUCTION}

Aerodynamic shape design is the main step of aircraft design and the airfoil optimization design is the key of the aerodynamic shape design. The traditional method of airfoil design is using personnel experience, wind tunnel test and flight test again and again to improve the optimization. However, long cycle high technical personnel and cost requirements limit application in practice of the method. With the rapid development of computer technology, computation fluid dynamics (CFD) is more and more mature. As a result, it is becoming more and more popular to adopt CFD technology in the airfoil optimization [1-3]. In the process of the airfoil optimization, algorithm is a key factor to decide the computation time which makes the algorithm to be important. Commonly used optimization algorithm is mainly divided into two categories: gradient optimization algorithm and global optimization algorithm. Although gradient optimization algorithm has low computing complexity, it is easy to fall into local optimal solution which has a bad effect on optimization. Meanwhile, global optimization algorithm has a strong search capability and is widely used [4-8], but the slow convergence speed and large amount of calculation is its shortcomings. Therefore, it becomes an important research topic that reduces solving times on the premise of guarantee calculation precision. Xia Lu et al. [9] reduce the computational by establishing the surrogate model of the flow field calculation. Bai Junqiang et al. [10] reduce the number of calculations by improving the optimization algorithm. In order to solve this problem, this paper proposes an algorithm to convert the global optimization algorithm and gradient algorithm. Based on the Isight optimization design platform, the airfoil optimization system is built to verify the feasibility of the combined optimization strategy.

\section{ORGANIZATION OF THE TEXT}

\section{Combined optimization strategy}

The combined optimization strategy uses global optimization algorithm firstly and then adopts gradient optimization algorithm to solve problem when the results converge to a certain precision. By this way, it not only gives full play to the advantages of the global optimization algorithm in the overall design space, but also has the gradient method's advantages which has a high efficiency in the local optimization. 


\subsection{Multi-Island Genetic Algorithm}

Genetic algorithms (GA) are search optimization methods that use principles of nature genetics and natural selections. In such methods, the solutions for a certain problem are represented by some form of biological population, which evolve over generations to adapt to an environment by selection, cross-over and mutation. Instead of working with a single solution at each iteration of the process, a GA works with a number of solutions, known as a population. GA has a good global searching ability. it is easy to appear premature phenomenon which leads to the convergence of the optimization results to the local optimal solution.

Multi-Island Genetic Algorithm (MIGA) is a non-classical optimization algorithm based on traditional genetic algorithm. It divides a large population into several subsets which is called the island vividly, as shown in figure 1 . In this method, the individuals in the island are exchanged in a certain proportion at regular intervals. In this way, it ensures the diversity of the population, and effectively suppresses the occurrence of premature phenomenon.

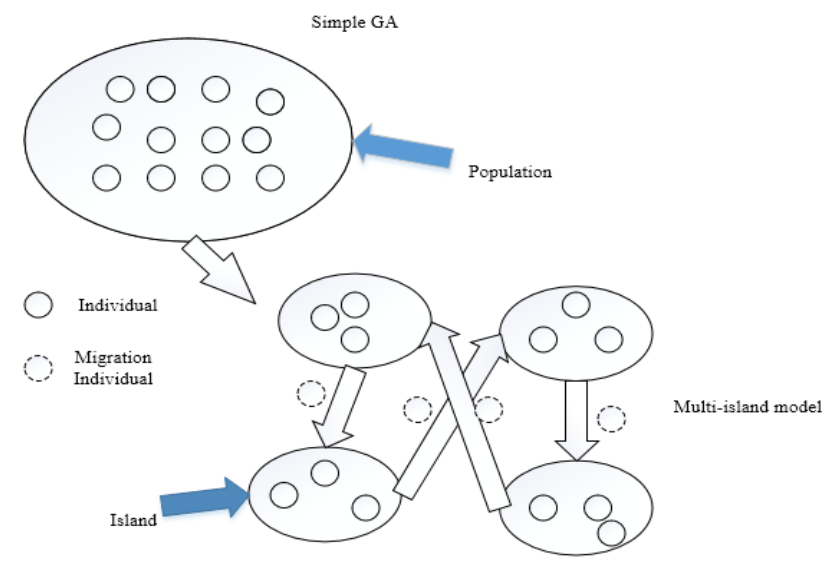

FIGURE 1. The evolutionary process of MIGA.

\subsection{Sequential Quadratic Programming}

The basic idea of Sequential Quadratic Programming (SQP) is to construct a Quadratic Programming (QP) sub problem to approximate original constrained optimization problem. By solving the QP sub problem, it can get an improved iterative point for the constrained optimization problems. There-fore, by repeating this process, we can get the iterative point which satisfies certain precision.

The general constrained optimization problem can be described as follows:

$$
\left.\begin{array}{ll}
\min & f(x) \\
\text { s.t. } & \mathrm{g}_{i}(x) \geq 0, i=1,2, \cdots \\
& h_{j}(x)=0, j=1,2, \cdots
\end{array}\right\}
$$

Based on the Taylor expansion to construct the QP sub problem is constructed at the current iteration point.

$$
\left.\begin{array}{r}
\min f(x)=\frac{1}{2} d^{T} \nabla^{2} f\left(x_{k} d\right)+\nabla f\left(x_{k}\right)^{T} d \\
\text { s.t. } g_{i}\left(x_{k}\right)+\nabla g_{i}\left(x_{k}\right)^{T} d \geq 0, i=1,2, \cdots \\
h_{j}\left(x_{k}\right)+\nabla h_{j}\left(x_{k}\right)^{T} d \geq 0, i=1,2, \cdots
\end{array}\right\}
$$

In Eq.(2), $d=x-x_{k}$. By solving equation (2), the optimal solution is obtained and new iteration point is $x_{k+1}=x_{k}+d_{k}$.

\subsection{Transformation Strategy}

When the global optimization algorithm is converted to the gradient optimization algorithm, it is necessary to set the transformation strategy to guarantee the global convergence. Therefore, the 
Algorithm transformation strategy (ATS) is proposed as follows:

It is assumed that after $n$ generations of population evolution the optimal solution is $x^{*}$, and the optimal solution of the $k$ generation of the population is $x_{k}$. Set $d(x)$ equal to the distance of individual value $x$ to $x^{*}$, and $\mathrm{D}$ is the distance from the initial solution to the optimal solution.

$$
\begin{gathered}
\Delta d\left(x_{k}\right)=d\left(x_{k+1}\right)-d\left(x_{k}\right) \\
y\left(x_{k}\right)=\frac{\Delta d\left(x_{k}\right)}{D}
\end{gathered}
$$

$\Delta d\left(x_{k}\right)$ is the difference between the optimal solutions of the two adjacent generations, and $y\left(x_{k}\right)$ is obtained by normalizing it.

$$
\begin{gathered}
\lambda \sum_{i=k}^{n-1} y\left(x_{k}\right)<\eta \\
\lambda \sqrt{\sum_{i=k}^{n-1}\left(y\left(x_{k}\right)-\lambda \sum_{i-k}^{n-1} y\left(x_{k}\right)\right)^{2}}<\sigma \\
\lambda=\frac{1}{n-k}
\end{gathered}
$$

The $k, \eta$ and $\sigma$ in the formula represents the adjustable parameters. $k$ value determines the population algebra involved in convergent budget. $\eta$ can constrain the average size of $\Delta d\left(x_{k}\right)$ and the $\sigma$ limits the degree of dispersion of the convergence process. Moreover, $\eta$ and $\sigma$ control convergence accuracy. When $y\left(x_{k}\right)$ satisfies the condition of the formula 5 and 6 , the algorithm carries on the transformation. By setting reasonable parameters, the algorithm can be converted to reduce the computation complexity of the whole problem on the premise of ensuring global convergence.

\subsection{Optimization Process}

Based on the combination optimization strategy optimization process shown in figure 2.

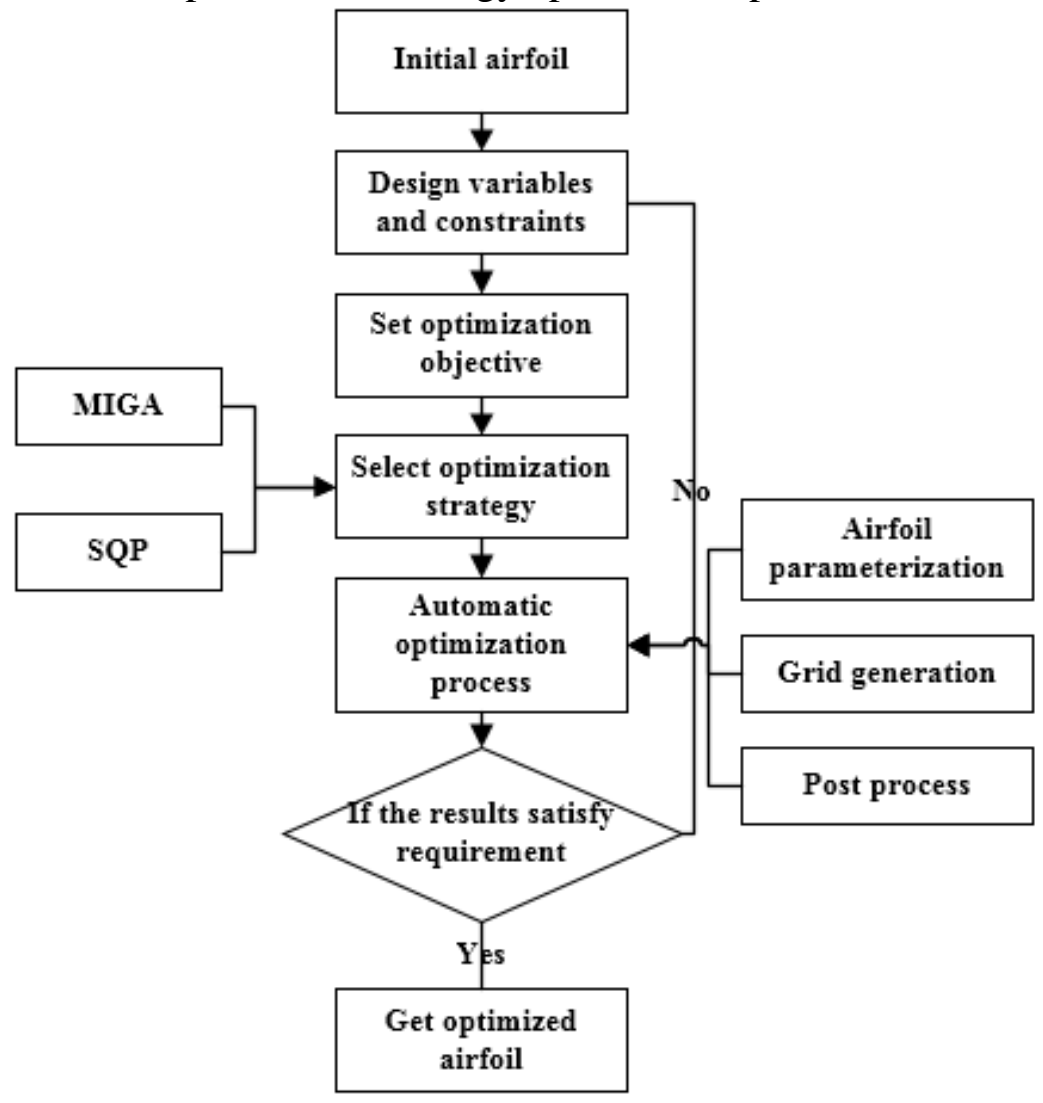

FIGURE 2. optimization design process

\section{Optimization Model}




\subsection{Airfoil Parameterization}

The parameterization of airfoil geometry has an important influence on the results of optimization design. At present, the commonly used parametric methods are as follows: Parsec airfoils, class shape transformations (CST) and the linear perturbation method [10]. The most commonly used method is Hicks-Henne shape function linear perturbation method [11-12].

The airfoil shape is a linear combination of the reference airfoil and the perturbation function as follows.

$$
y(x)=y_{\text {basic }}(x)+\sum_{i=1}^{\mathrm{n}} a_{i} f_{i}
$$

Where $y_{\text {bacic }}(x)$ are the ordinates of the surface of the baseline sections and fi are the shape functions shown in Eq. (9) and Eq. (10). $a_{i}$ associate with the function which determined by the value of the participation coefficients. $\mathrm{y}(\mathrm{x})$ are the longitudinal coordinates after the disturbance of the reference airfoil.

$$
\begin{gathered}
f_{i}(x)= \begin{cases}x^{0.5}(1-x) \mathrm{e}^{-15 x} & (i=1) \\
\sin ^{3}\left(\pi x^{e(k)}\right) & (i>1)\end{cases} \\
e(k)=\frac{\lg 0.5}{\lg k}(0<k<1)
\end{gathered}
$$

When $\mathrm{i}>1$, the derivative of the shape function $f(x)$ are as follows.

$$
\begin{gathered}
f_{x}^{\prime}=3 \sin ^{2}\left(\pi x^{e(k)}\right) \cos \left(\pi x^{e(k)}\right) e(k) x^{e(k)-1} \\
e(k)=\frac{\lg 0.5}{\lg k}, 0<k<1
\end{gathered}
$$

Obviously, at the time $x=1, f(x)=0, f^{\prime}(x)=0$, that is, Hicks-Henne shape function and its derivate function is 0 when $x=1$.This means that the shape function near the trailing edge is 0 .That is the trailing edge of the base airfoil is substantially free of disturbance. However, the trailing edge angle is an important parameter that affects the aerodynamic characteristics of airfoil. What's more, the disturbance of the original Hicks-Henne type function to the trailing edge has a direct impact on the optimization process. Accordingly, the $\mathrm{H}$ function is improved and expressed by the following equation.

$$
\begin{gathered}
f(x)= \begin{cases}x^{0.5}(1-x) \mathrm{e}^{-15 x} & (i=1) \\
\sin ^{3}\left(\pi x^{e(k)}\right) & (1<i<n-2) \\
\sin ^{3}\left(\pi(1-x)^{e(1-k)}\right) & (i=n-1) \\
10 x(1-x) \mathrm{e}^{-10(1-x)} & (i=n)\end{cases} \\
\quad e(k)=\frac{\lg 0.5}{\lg k}, 0<k<1
\end{gathered}
$$

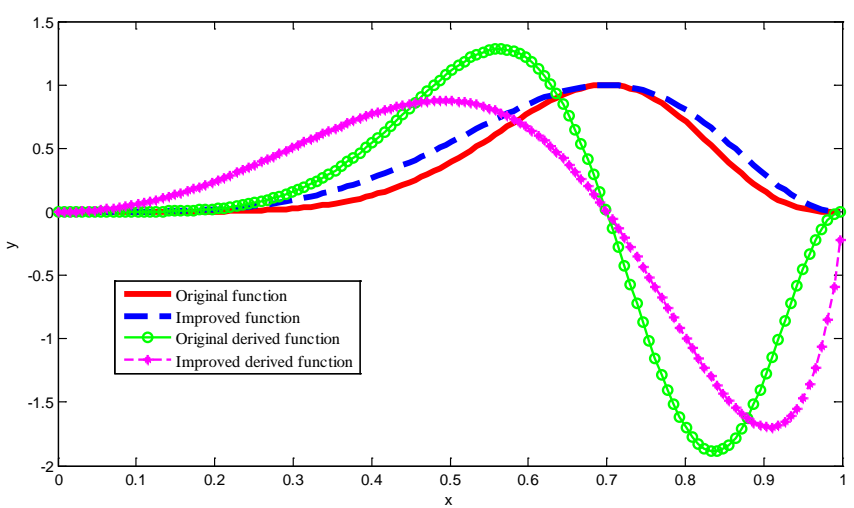

FIGURE 3. The comparison of original and improved Hicks-Henne shape function 


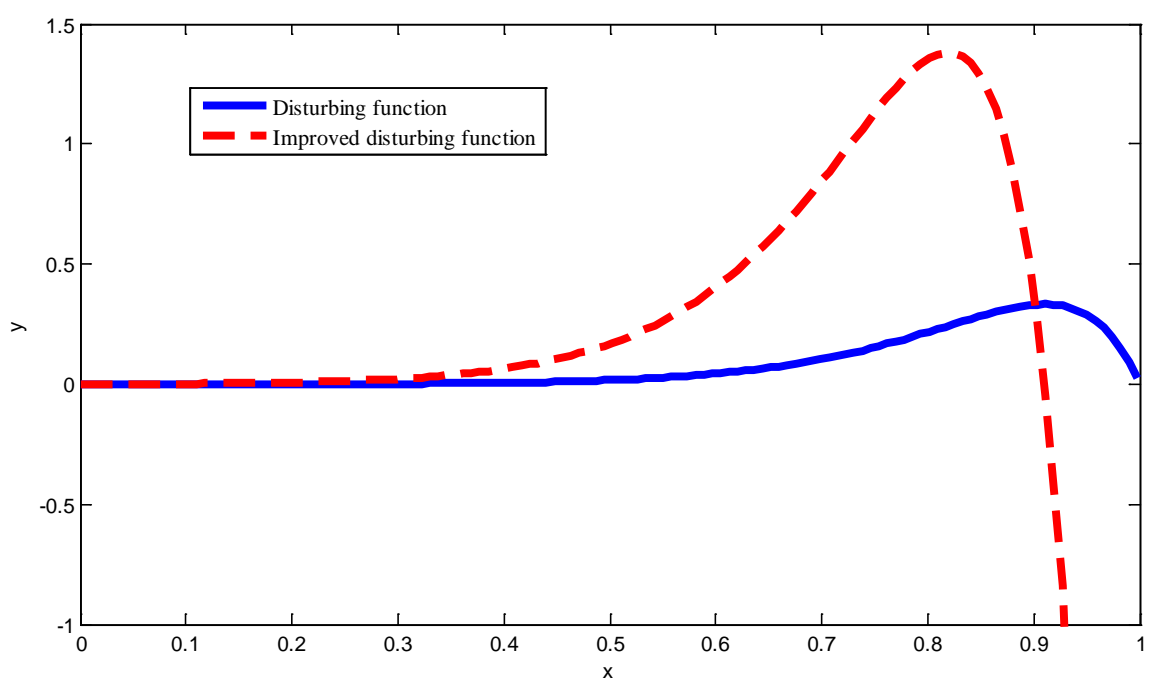

FIGURE 4. The diagrams of trailing edge’s Hicks-Henne shape function

Figure 3 shows simulation comparison of original and improved Hicks-Henne shape functions when $i=n-1$ and $k=0.7$. The improved shape function is slow attenuation on both sides of $x=k$, and its derivate function reaches a value other than 0 when $x$ close to 1 . This will help the shape function's disturbance to the rear of the airfoil. Figure 4 shows the improved Hicks-Henne shape function increases the disturbance range of trailing edge which is helpful to the development of the optimization process.

\subsection{Flow Field Calculation Method}

Airfoil uses ICEM-CFD scripting language for $C$ type mesh. It is necessary to set a reasonable $\mathrm{y}^{+}$value and closed wall size for ensuring the accuracy of the results. The aerodynamic analysis uses Navier-Stokes control equation to calculate the airfoil flow field by Fluent software. Turbulence model applies the shear stress transmission (SST) $k-w$ model. The boundary is set as the pressure field and second order upwind difference scheme is used to discretize the control model.

\subsection{Example Verification}

In order to verify the rationality of the calculation of the flow field, the RAE2822 is chosen as the validation airfoil. The design states as follows: Mach number is 0.74 , Angle of attack is 2.31 degrees and Reynolds number is $6.5 \times 106$. The calculated data agree well with the experimental data [13], which verifies the rationality of the flow field model.

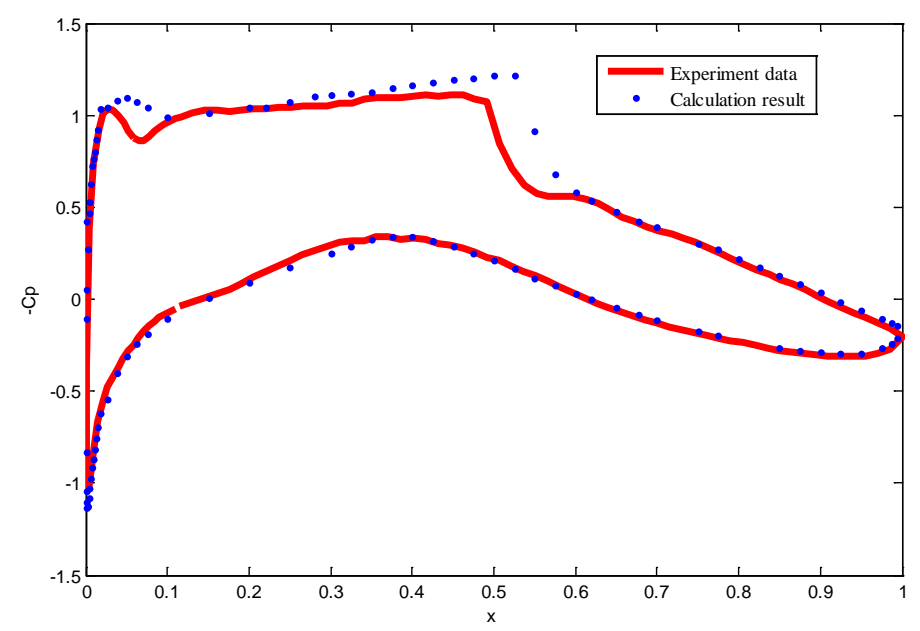

FIGURE 5. The comparison of computational data and experimental data 


\section{Airfoil Optimization Example}

\subsection{Variable Design}

Taking the RAE2822 airfoil as the reference airfoil, the research object is its subsonic cruise performance at $10 \mathrm{~km}$ altitude. 14 design variables are selected to control the disturbance, and the upper and the lower wing surface of the airfoil are divided into 7 . What's more, $\mathrm{k}$ take $0.10,0.26$, $0.42,0.58,0.74$ and 0.90 , and the corresponding i value are 2, 3, 4, 5, 6 and 7 . Calculations consider a single flight condition, that is, freestream Mach number 0.74 , freestream Reynolds number $6.36 \times$ 106,the angle of attack is 2.3 degrees. The expression of the optimization problem is shown in Eq. (15).

$$
\left.\begin{array}{ll}
\max & f(x)=C_{L} / C_{D} \\
\text { s.t. } & C_{L} \geq C_{L 0} \\
& d_{\max } \geq 0.9 d_{\max 0}
\end{array}\right\}
$$

In Eq. (15), $C_{L 0}$ is the initial airfoil lift coefficient and $d_{\max 0}$ is the initial maximum thickness of the airfoil.

\subsection{Result Analysis}

The parameters of two kinds of optimization algorithms are as follows.

Optimization algorithm 1: MIGA is used in optimization plan, and the parameters of the MIGA are as follows: sub-population size 12 , number of island 4 , number of generations 30 , rate of crossover 0.8 , rate of mutation 0.03 , rate of migration 0.4 and interval of migration 4 .

Optimization algorithm 2: the setting of MIGA is the same with optimization algorithm 1, the parameters of SQP are as follows: the maximum number of iterations is set to 200, and the convergence precision is set to 1 . What's more, ATS transformation strategy is adopted, and the parameters setting are as follows: $\mathrm{k}=\mathrm{n}-2, \eta=0.03, \sigma=0.01$.

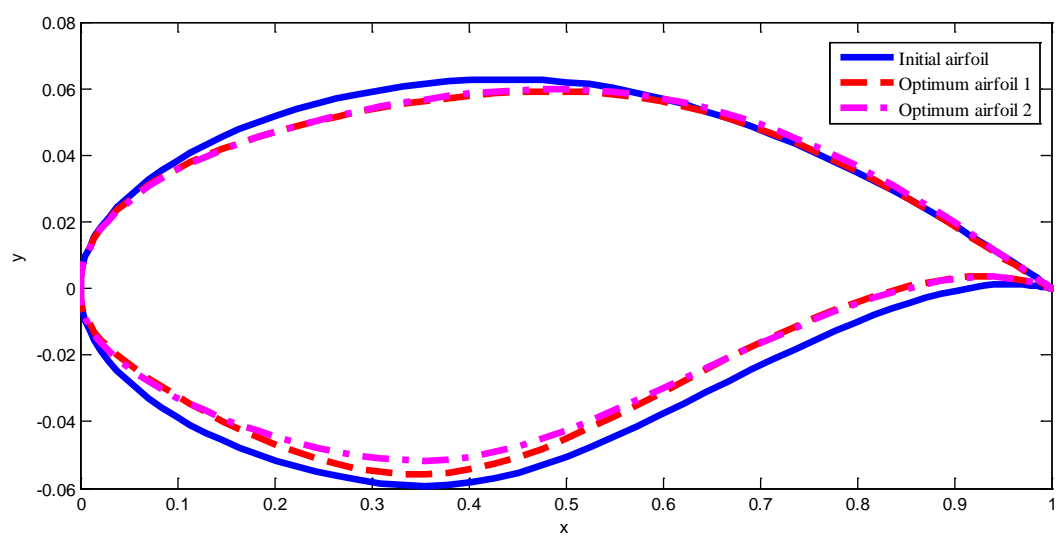

FIGURE 6. The comparison of airfoil shape 


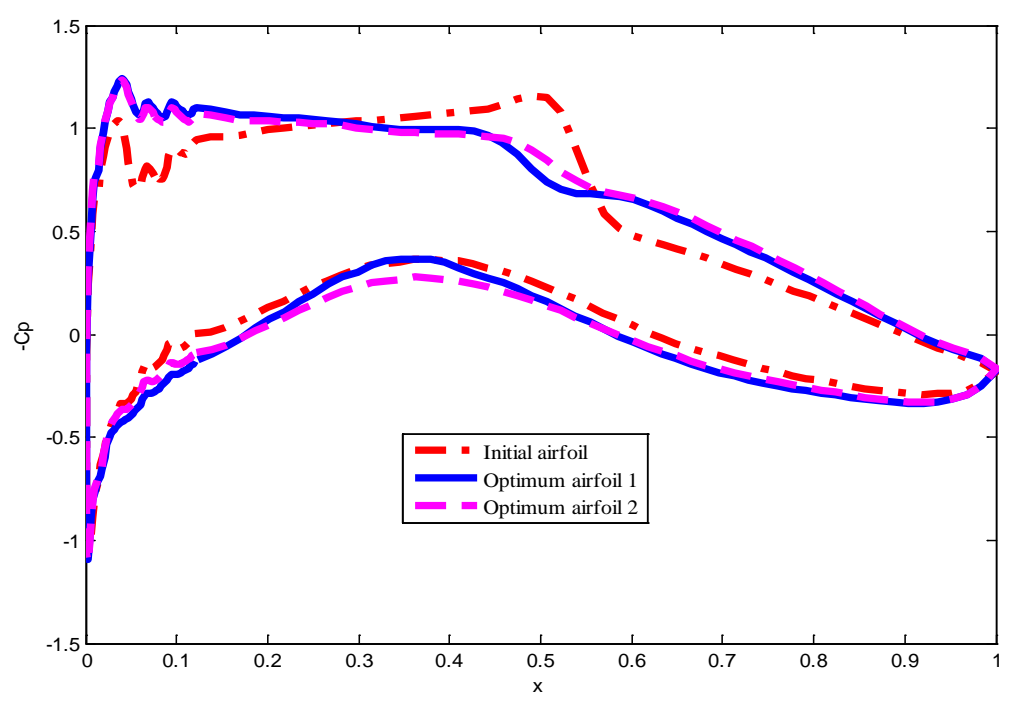

FIGURE 7. The comparison of pressure coefficient

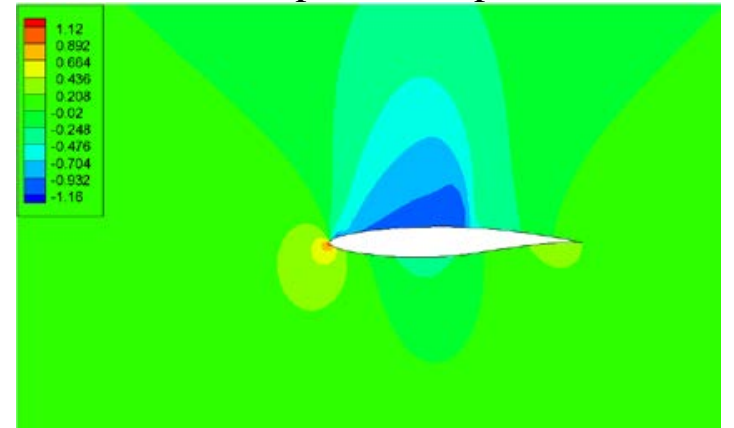

(a) Initial airfoil

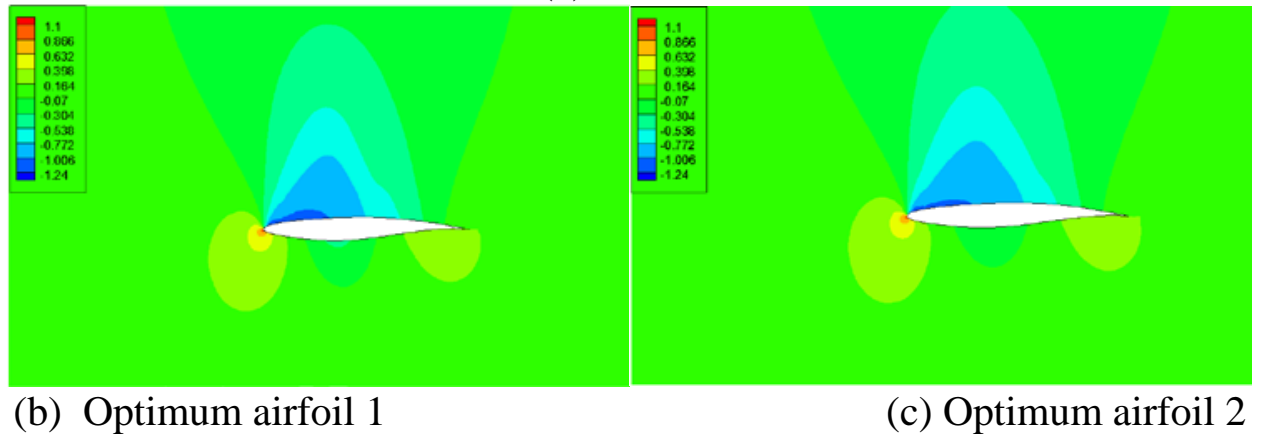

FIGURE 8. The comparison of pressure coefficient cloud picture 
Table 1. The comparison of optimization performance

\begin{tabular}{|c|c|c|c|c|c|}
\hline & $d_{\max }$ & $\mathbf{C}_{\mathbf{L}}$ & $C_{D}$ & $\begin{array}{l}\text { Lift drag } \\
\text { ratio }\end{array}$ & $\begin{array}{c}\text { Percentage } \\
\text { increase }\end{array}$ \\
\hline Initial airfoil & 0.121 & 0.643 & 0.016 & $6^{39.82}$ & 1 \\
\hline $\begin{array}{l}\text { Optimum } \\
\text { airfoil } 1\end{array}$ & 0.113 & 0.736 & 0.014 & $5^{51.14}$ & 28.421 \\
\hline $\begin{array}{l}\text { Optimum } \\
\text { airfoil } 2\end{array}$ & 0.109 & 0.750 & 0.014 & $1^{51.99}$ & 30.545 \\
\hline
\end{tabular}

Figure 7 shows the comparison of the airfoil profile before and after optimization. Two optimized airfoil shapes are similar which shows that the two optimization algorithms converge to the optimal solution. Compared with the baseline airfoil, the optimized airfoils become thinner and leading edge radius become smaller. Thus the airfoil resistance is reduced. Figure 8 and 9 shows that the suction peak value of the leading edge of the airfoil becomes larger, and the pressure value on the airfoil surface decreases after optimization. Shock move forward and is reduced.

Table 1 shows the comparison of optimization performance. After optimization, $\mathrm{C}_{\mathrm{L}}$ was increased by $14.391 \%$ and $16.670 \%$, while $C_{D}$ was decreased by $10.898 \%$ and $10.216 \%$. As a result, the lift-drag ratio is increased by $28.421 \%$ and $30.545 \%$. The optimization algorithm 1 called the flow field calculation model 1 times. Meanwhile, the optimization algorithm 2 triggered ATS after 20 generations of population evolution, and the SQP algorithm iterated 120 times. This means that the optimization algorithm 2 called the flow field calculation model 1 times. Compared with the optimization algorithm 1, the optimization algorithm 2 improved the performance of the algorithm by $2.124 \%$, and the number of calculation decreased by $28.4 \%$. Based on the result above, the combined optimization strategy has excellent global optimization and rapid convergence.

\section{CONCLUSION}

The Hicks-Henne shape function is improved which lead the tail thickness and the trailing edge angle can be adjusted more conveniently. Therefore, the design space of its parameters is increased on the premise of ensuring the smooth curve of the airfoil.

In order to optimize the algorithm combination, a transformation strategy is proposed to control the conversion of two optimization algorithms. This strategy is a general strategy which can be applied to other algorithms.

Based on the Isight optimization design platform, the optimization system module is built by the combination optimization strategy. The results show that the combination optimization strategy has good ability of searching in the aspect of calculation precision and computing time and the whole CFD calculation time is reduced.

\section{REFERENCES}

[1] WANG B. Numerical Simulations and Optimization on Aerodynamic Characteristics of Advanced Rotor by CFD Method[D]. Nanjing University of Aeronautics and Astronautics, 2012.

[2] ZHAO K. Complex Aerodynamic Optimization and Robust Design Method Based on Computational Fluid Dynamics[D]. Xi An: Northwestern Polytechnical University, 2015.

[3] WAN T H, ZHAO D L, LIANG W K. Aerofoil Optimization of Fluid Machinery Based on CAD/CFD system [J]. Fluid Machinery ,2005,33(11):37-39

[4] WANG X P. Study of genetic algorithm for aerodynamic optimization and design[J]. Acta Aerodynamica Sinica, 2001, 19(2):129-134.

[5] Antunes A P, Azevedo J L F. An aerodynamic optimization computational framework using genetic algorithms[J]. Journal of the Brazilian Society of Mechanical Sciences and Engineering, 2016, 38(4): 1037-1058.

[6] Morris A, Allen C, Rendall T. Wing Design by Aerodynamic and Aeroelastic Shape 
Optimisation[M]// 26th Applied Aerodynamics Conference. 2013.

[7] Nakamura M, Izui K, Nishiwaki S, et al. A Multi-Objective Particle Swarm Optimization Incorporating Design Sensitivities[C]// Aiaa/issmo Multidisciplinary Analysis and Optimization Conference. 2006.

[8] Sun R J, ZHAN H.Synthesis Airfoil Optimization by Particle Swarm Optimization Based on Global Information[J].Acta Aeronautica et Astronautica Sinica . 2010 (11): 2166-2173.

[9] XIA L, WANG D, ZHANG Y.Aerodynamic optimization method based on adaptive surrogate model. Acta Aerodynamica Sinica,,2016,34(4):433-440.

[10] LIAO Y P, LIU L, LONG T. The Research on Some Para meterized Methods for Airfoil[J]. Journal of Projectiles, Rockets, Missiles and Guidance, 2011, 31 (3) : 160-164. ( in Chinese)

[11] XU P, JIANG C S. Optimization Design of Laminar Aerofoil Based on Genetic Algorithms and Hicks-Henne Shape Function[J]. Journal of Air Force Engineering University (Natural Science Edition), 2009, 10(1):13-16. (in Chinese)

[12] CHEN X K, GUO Z, YI F, et al. Aerodynamic shape optimization and design of airfoils with low Reynolds number[J]. Acta Aeronautica et Astronautica Sinica, 2014(3):300-307. (in Chinese)

[13] Cook, P.H., M.A. McDonald, M.C.P. Firmin, "Aerofoil RAE 2822 - Pressure Distributions, and Boundary Layer and Wake Measurements," Experimental Data Base for Computer Program Assessment, AGARD Report AR 138, 1979. 